\title{
Bound States in Delta Function Potentials
}

\author{
Sydney Geltman \\ Department of Physics, University of Colorado, Boulder, CO 80309, USA \\ Correspondence should be addressed to Sydney Geltman, geltman@colorado.edu
}

Received 28 June 2011; Accepted 18 September 2011

Academic Editor: Gregory Lapicki

Copyright ( 2011 Sydney Geltman. This is an open access article distributed under the Creative Commons Attribution License, which permits unrestricted use, distribution, and reproduction in any medium, provided the original work is properly cited.

We present a systematic treatment of the bound state structure of a short-range attractive interatomic potential in one, two, and three dimensions as its range approaches zero. This allows the evaluation of the utility of delta function potentials in the modeling of few-body systems such as nuclei, atoms, and clusters. The relation to scattering by delta function potentials is also discussed.

\section{Introduction}

The delta function has played an enormous role in the development and advancement of quantum mechanics since its introduction by Dirac [1]. From its early use in the momentum representation in scattering theory, it has found many conceptual applications. In the present work, we study its applicability to represent a very short-ranged attractive interatomic potential and what bound state structure may be expected in the zero-range limit.

In 1935, Thomas [2] published a paper in which he investigated the possible relationship between the mass defect of the deuteron and the range of the proton-neutron force. The conclusion was that a finite mass defect, attributable to a finite binding energy, was not consistent with a zerorange potential. He showed that going to the limit of a zero range proton-neutron attractive potential could only result in an infinite binding energy, or infinite mass defect, which contradicts the experimental finding of a finite mass defect. An extension of this argument to the triton would conclude that, in the limit of zero-range two-body forces, the threebody system would also tend to have an infinite binding energy. This suggests that the use of delta function potentials may lead to unrealistic binding energies in composite systems. Our present objective is to treat the problem in a more complete and systematic manner by evaluating all the bound states rather than using a variational method that only gives an upper bound to the ground state energy [2].

In more recent years, delta function potentials have been used to represent model atomic systems in their interactions with electromagnetic fields, that is, in multiphoton absorption or ionization processes. While the above difficulties that arose in the nuclear case would also be present in the threedimensional treatment of electron binding in an atom, a feasible situation presents itself in the one-dimensional case, where the existence of a single bound state may serve as a reasonable model for the ground state of an atom, or an even better model for a negative ion, which typically has only one bound state. For linearly polarized radiation fields, the use of a one-dimensional atomic model is also physically reasonable and has been extensively used [3-7].

Another area where delta function potentials have come into considerable use is in the description of a many-atom system, such as a cluster or a Bose-Einstein condensate. If the atomic density is small enough such that the mean interatomic separation is large compared to the range of the interatomic force or potential, it is reasonable to represent the interatomic potential by a weighted delta function, $-B \delta(\mathbf{r}-$ $\mathbf{r}^{\prime}$ ). This often arises where a many-body problem is treated in a mean-central-field approximation and enters into the Hartree self-consistent-field nonlinear wave equation as

$$
\int d \mathbf{r}^{\prime} V\left(\mathbf{r}-\mathbf{r}^{\prime}\right)\left|\Psi\left(\mathbf{r}^{\prime}\right)\right|^{2} \cong-B|\Psi(\mathbf{r})|^{2} .
$$

This is a reasonable way of handling the interatomic potential in a diffuse many-body system and does not depend on the bound states in the zero-range limit. However, the evaluation of the coefficient $B$ (often called $U_{0}$ in the literature) is not obvious and has been the subject of some disagreement [8-11]. 
As is well known, square well potentials have been used extensively to model bound-state systems since the beginning of quantum mechanics and are discussed in practically every text on it.

Their generalization to an arbitrary number of spatial dimensions has also been carried out $[12,13]$. Our purpose in this paper is to present a unified picture of how by letting the range of the square well approach zero in one, two, and three dimensions, one may deduce the bound-state structure in the delta function limit.

We will approach the problem for delta function potentials in one, two, and three dimensions in the following sections. These potentials are denoted as

$$
V_{j}(\mathbf{R})=-B_{j} \delta(\mathbf{R}),
$$

where $B_{j}(j=1,2,3)>0$. The strength coefficient $B_{j}$ will have varying dimensionalities, since every $V_{j}$ must have the dimensions of energy, while each $\delta(\mathbf{R})$ has the dimensionality of inverse volume, since $\int d \mathbf{R} \delta(\mathbf{R})=1$. We will take the $\delta(\mathbf{R})$ 's for each dimensionality to be the limit of a step function,

$$
\begin{gathered}
\text { 1D: } \delta(x)= \begin{cases}\lim _{x_{0} \rightarrow 0}\left(\frac{1}{2 x_{0}}\right), & |x| \leq x_{0}, \\
0, & |x|>x_{0},\end{cases} \\
\text { 2D: } \delta(\rho, \varphi)= \begin{cases}\left(\frac{1}{2 \pi}\right) \lim _{\rho_{0} \rightarrow 0}\left(\frac{2}{\rho_{0}^{2}}\right), & \rho \leq \rho_{0}, \\
0, & \rho>\rho_{0},\end{cases} \\
\text { 3D: } \delta(r, \vartheta, \varphi)= \begin{cases}\left(\frac{1}{4 \pi}\right) \lim _{r_{0} \rightarrow 0}\left(\frac{3}{r_{0}^{3}}\right), & r \leq r_{0}, \\
0, & r>r_{0} .\end{cases}
\end{gathered}
$$

Since $-B_{j}<0$, our extended potentials will be attractive square wells. We first evaluate the bound states for the extended square well and then go to the limit of zero range. The symmetries that arise in each dimensionality for the assumed square wells and the corresponding wave function separabilities are

1D: even, $\Psi_{g}(-x)=\Psi_{g}(x)$, and odd, $\Psi_{n}(-x)=-\Psi_{u}(x)$,

2D: $\Psi(\boldsymbol{\rho})=\chi_{m}(\rho) e^{i m \varphi}$,

3D: $\Psi(\mathbf{r})=R_{l}(r) Y_{l m}(\theta, \phi)$.

\section{One Dimensional}

The Schrödinger equation (atomic units used throughout) for the above assumed $1 \mathrm{D}$ potential is

$$
\left[-\left(\frac{1}{2 M}\right)\left(\frac{d^{2}}{d x^{2}}\right)+V_{1}(x)-E\right] \Psi(x)=0,
$$

where $M$ is the reduced mass and

$$
V_{1}(x)= \begin{cases}-\frac{B_{1}}{2 x_{0}}, & |x| \leq x_{0} \\ 0, & |x|>x_{0}\end{cases}
$$

The two allowed symmetries are $\Psi_{g}$ (even), that is, $\cos \left(k_{i} x\right)$, and $\Psi_{u}$ (odd), that is, $\sin \left(k_{i} x\right)$ for $|x|<x_{0}$, where $k_{i}=$ $\sqrt{2 M\left(B_{i} / 2 x_{0}-|E|\right)}$ inside the well. Outside the potential well, both $\Psi_{g}$ and $\Psi_{u}$ have the form for $|x|>0, e^{i k_{0}|x|}$, where $k_{0}=i \sqrt{2 M|E|}$. Joining inside and outside solutions at $|x|=x_{0}$ with continuous $\Psi^{\prime} / \Psi$ gives the transcendental equations for $|E|(E<0$ for bound state),

$$
\begin{array}{ll}
\text { even: } \quad \tan \left(k_{i} x_{0}\right) & =\frac{\left|k_{0}\right|}{k_{i}}, \\
\text { odd: } \quad \tan \left(k_{i} x_{0}\right) & =-\frac{k_{i}}{\left|k_{0}\right|} .
\end{array}
$$

We now want to use these to find the bound state energies in the $x_{0} \rightarrow 0$ limit, that is, for the delta function potential. In going to this limit, we note that $k_{i} x_{0}=\sqrt{M B_{1} x_{0}(1-f)}$, where $f=|E| /\left(B_{1} / 2 x_{0}\right)$ is the binding energy expressed as a fraction of the well depth. Since $k_{i} x_{0} \rightarrow 0$ as $x_{0} \rightarrow 0$, we may expand $\tan \left(k_{i} x_{0}\right) \rightarrow k_{i} x_{0}+\vartheta\left(\left(k_{i} x_{0}\right)^{3}\right)$. For the even case, this leads to $E \rightarrow-M B_{1}^{2} / 2$ as the only bound state in the limit.

The situation is somewhat more complicated for the odd symmetry. Again expanding $\tan \left(k_{i} x_{0}\right)$ and dropping $\vartheta\left(\left(k_{i} x_{0}\right)^{3}\right)$, we have

$$
E \underset{x_{0} \rightarrow 0}{\longrightarrow} \frac{-1}{\left(2 M x_{0}^{2}\right)}
$$

For this to be an acceptable bound state energy, its absolute value must lie within the range of the well depth, or $B_{1} /\left(2 x_{0}\right)>|E|>0$. Thus, the minimum value of $x_{0}$ that can support a bound state of odd symmetry is approximately $1 /\left(M B_{1}\right)$, and there are no bound states for lower $x_{0}$, which of course includes the delta function limit.

For large enough $x_{0}$, there may be many even and odd symmetry bound states since the singularities of the tangent functions will give a number of intersections with the rhs of (7), in fact there will be about $\sqrt{M B_{1} x_{0}} / \pi$ such intersections.

\section{Two Dimensional}

Adopting the polar coordinates $\rho, \varphi$ in 2D space, the Schrödinger equation is

$\left[-\left(\frac{1}{2 M}\right)\left\{\frac{\partial^{2}}{\partial \rho^{2}}+\frac{1}{\rho} \frac{\partial}{\partial \rho}+\frac{1}{\rho^{2}} \frac{\partial^{2}}{\partial \phi^{2}}\right\}+V_{2}(\rho)-E\right] \Psi(\rho, \varphi)=0$,

where $V_{2}(\rho)$, from (2) and (3), is

$$
V_{2}(\rho)= \begin{cases}-\frac{B_{2}}{\pi \rho_{0}^{2}}, & \rho \leq \rho_{0} \\ 0, & \rho>\rho_{0}\end{cases}
$$

This central potential allows the separability, $\Psi(\rho, \varphi)=$ $\chi_{m}(\rho) e^{i m \varphi}$, where $\chi_{m}(\rho)$ satisfies the radial equation

$$
\left[\frac{d^{2}}{d \rho^{2}}+\left(\frac{1}{\rho}\right) \frac{d}{d \rho}-\frac{m^{2}}{\rho^{2}}+k^{2}\right] \chi_{m}(\rho)=0,
$$


and the inside and outside $k^{\prime}$ s take the values

$$
\begin{gathered}
k_{i}=\left[2 M\left(\frac{B_{2}}{\pi \rho_{0}^{2}}-|E|\right)\right]^{1 / 2}, \quad \rho \leq \rho_{0}, \\
k_{0}=i(2 M|E|)^{1 / 2}, \quad \rho>\rho_{0} .
\end{gathered}
$$

These are Bessel equations, and we must join the inner regular solution $\chi_{m}(\rho)=J_{m}\left(k_{i} \rho\right)$ to the outer decaying solution, $K_{m}\left(\left|k_{0}\right| \rho\right)$ the modified Bessel function [14]. We restrict our present calculation to the case of zero angular momentum, that is, $m=0$, the one having the largest number of bound states.

We first note that the arguments of the Bessel functions at the edge of the well are

$$
\begin{gathered}
k_{i} \rho_{0}=\left[\frac{2 M B_{2}}{\pi}\left(1-\frac{|E|}{\left(B_{2} / \pi \rho_{0}^{2}\right)}\right)\right]^{1 / 2}, \\
\left|k_{0}\right| \rho_{0}=\left[\frac{2 M B_{2}}{\pi} \frac{|E|}{\left(B_{2} / \pi \rho_{0}^{2}\right)}\right]^{1 / 2} .
\end{gathered}
$$

Since the depth of the potential well (from (3)) is $B_{2} / \pi \rho_{0}^{2}$, we may define the bound states, $E=-|E|$, as their fractional amount of the potential depth (measured from the top of the well where $E=0$ ). The only free variable remaining is the product $M B_{2}$. We may then obtain numerically the roots for the continuity of logarithmic derivative at $\rho_{0}$,

$$
\frac{k_{i} J_{0}^{\prime}\left(k_{i} \rho_{0}\right)}{J_{0}\left(k_{i} \rho_{0}\right)}=\frac{\left|k_{0}\right| K_{0}^{\prime}\left(\left|k_{0}\right| \rho_{0}\right)}{K_{0}\left(\left|k_{0}\right| \rho_{0}\right)},
$$

to give all the roots for $|E| /\left(B_{2} / \pi \rho_{0}^{2}\right)$ as a function of $M B_{2}$.

Again, a good estimate of the number of bound states is given by $\sqrt{2 M B_{2} / \pi}$ divided by $\pi$ since asymptotically $J_{0}(z)=$ $\cos (z-\pi / 4) / \sqrt{z}$.

Thus, the number of bound states increases as $\sqrt{M B_{2}}$, while remaining independent of $\rho_{0}$. The absolute values of each of these bound states will $\rightarrow \infty$ as $\rho_{0} \rightarrow 0$ since the potential depth is increasing as $B_{2} / \pi \rho_{0}^{2}$. This differs markedly from the 1D case where both the number of bound states and their relative and absolute values also depended on $x_{0}$.

\section{Three Dimensional}

The Schrödinger equation in the 3D spherical coordinates $r, \theta, \varphi$ is

$$
\begin{aligned}
{\left[-\left(\frac{1}{2 M}\right)\left\{\frac{\partial^{2}}{\partial r^{2}}+\frac{2}{r} \frac{\partial}{\partial r}+\frac{1}{r^{2} \sin \theta} \frac{\partial}{\partial \theta}\left(\sin \theta \frac{\partial}{\partial \theta}\right)\right.\right.} \\
\left.\left.+\frac{1}{r^{2} \sin ^{2} \theta} \frac{\partial^{2}}{\partial \varphi^{2}}\right\}+V_{3}(r)-E\right] \Psi(r, \theta, \varphi)=0,
\end{aligned}
$$

where $V_{3}(r)$ is given in (2) and (3). Again with separability, $\Psi(r, \theta, \varphi)=R_{l}(r) Y_{l m}(\theta, \varphi)$, where $R_{l}(r)$ satisfies the radial wave equation

$$
\left[\frac{d^{2}}{d r^{2}}+\left(\frac{1}{r}\right)\left(\frac{d}{d r}\right)-\frac{l(l+1)}{r^{2}}+k^{2}\right] R_{l}(r)=0,
$$

where $k$ takes the inside and outside values,

$$
\begin{gathered}
k_{i}=\left[2 M\left(\frac{3 B_{3}}{4 \pi r_{0}^{3}}-|E|\right)\right]^{1 / 2}, \quad r \leq r_{0}, \\
k_{0}=i(2 M|E|)^{1 / 2}, \quad r>r_{0} .
\end{gathered}
$$

As previously done, since higher angular momentum bound states will be fewer than those for $l=0$, we will restrict our attention to s-states.

The solutions of (15) are the spherical Bessel functions, of the first kind inside the potential well [14],

$$
R_{0}(r)=j_{0}\left(k_{i} r\right)=\frac{\sin \left(k_{i} r\right)}{k_{i} r},
$$

and modified (of the third kind) outside the well,

$$
R_{0}(r)=h_{0}^{(2)}\left(i k_{0} r\right)=\frac{e^{-\left|k_{0}\right| r}}{\left|k_{0}\right| r} .
$$

Demanding continuity of the logarithmic derivatives at $r_{0}$ gives the transcendental equation for the bound states in terms of their fractional amount of the well depth, $f=$ $|E| /\left(3 B_{3} / 4 \pi r_{0}^{3}\right)$,

$$
\tan \left[\sqrt{\frac{3 M B_{3}}{2 \pi r_{0}}}(1-f)\right]+\sqrt{\frac{(1-f)}{f}}=0 .
$$

Unlike the 2D case, the coefficient of $1-f$ in the tangent also depends on $r_{0}$, so the ratios between various bound state energies do not remain independent of $r_{0}$. Again, from the periodicity of the tangent function, we estimate the total number of bound states to be $\left(3 M B_{3} / 2 \pi r_{0}\right)^{1 / 2} / \pi$.

Thus, in the delta function limit, there are an infinite number of bound states, and they each approach infinite binding energy.

All of our present interest in the delta function potential has been in its bound state structure. It is well known, however, that there is a close relationship between the bound states and the scattering properties of a potential. This was first investigated by Fermi [15] for the delta function case in the study of the scattering of neutrons by hydrogen atoms. Zero energy scattering is characterized by the scattering length, defined as $=\lim _{k \rightarrow 0}\left(-\tan \eta_{0} / k\right)$, where $k=\sqrt{2 M E}, M$ is the reduced mass and $\eta_{0}$ is the s-wave phase shift. For the present $3 \mathrm{D}$ case, this may be evaluated exactly and is given by

$$
a=r_{0}-\left(\frac{r_{0}^{3 / 2}}{c}\right) \tan \left(c r_{0}^{-1 / 2}\right),
$$

where $c=\left(3 M B_{3} / 4 \pi\right)^{1 / 2}$. This is clearly indeterminate in the $r_{0} \rightarrow 0$ limit. It is further closely tied to our present result that the number of bound states becomes infinite in the delta function $r_{0} \rightarrow 0$ limit. The connection is Levinson's theorem [16] that states that $\eta_{0} \underset{\varepsilon \rightarrow 0}{\rightarrow} N_{0} \pi$, where $N_{0}$ is the number of bound s-states. Much theoretical work [17-21] has gone into the removal of this indeterminacy by a procedure known as regularization, requiring much 
more refined advanced mathematical considerations than are needed in the present bound state study. Another aspect of delta function potentials is that their detailed study is useful in many aspects of quantum field theory, and an excellent survey of this subject is contained in the paper of Gosdzinsky and Tarrach [22].

\section{Summary}

The objective of the present paper is to present a unified treatment for the one, two, and three dimensional cases that might have application to actual physical systems and look at their bound state structure as the potential well radius is shrunk to zero, thus approaching delta function potentials. We have shown that the bound state structure of attractive delta function potentials differ considerably depending on their dimensionality.

In the $1 \mathrm{D}$ case for the even symmetry, there is a single well defined bound state at $E=-M B_{1}^{2} / 2$, while there are none for the odd symmetry. This does not occur for the higher dimensionalities, where all bound energies become infinite in the zero range limit, and thus provides the unique opportunity to represent a model bound atomic system. Indeed, it has been used to investigate photoabsorption processes with linearly polarized radiation or tunnelling ionization due to a static electric field [3-7].

In the $2 \mathrm{D}$ case, there is a finite number of bound states, given approximately by $\left(2 M B_{2} / \pi^{3}\right)^{1 / 2}$, each of which grows as $1 / \rho_{0}^{2}$ as $\rho_{0} \rightarrow 0$, or each of them approaching infinite binding energy. This makes the $2 \mathrm{D}$ delta function potential also not usable to represent bound atomic systems, since the latter have finite binding energies.

In the $3 \mathrm{D}$ case, the delta function limit produces both an infinite number of states, $\sim\left(3 M B_{3} / 2 \pi^{3} r_{0}\right)^{1 / 2}$, as well as each state approaching infinite binding energy as $-B_{3} / r_{0}^{3}$. Thus, a $3 \mathrm{D}$ delta potential is also unsatisfactory to represent any stable two-body (or more) nuclear, atomic, or cluster system. This confirms the conclusion reached by Thomas [2] 76 years ago and hopefully provides a more complete understanding of it. The Thomas result was obtained variationally as an upper bound, and it referred only to the ground state, while our present treatment covers all the bound states.

\section{References}

[1] P. A. M. Dirac, The Principles of Quantum Mechanics, Oxford University Press, 3rd edition, 1940.

[2] L. H. Thomas, "The interaction between a neutron and a proton and the structure of H3," Physical Review, vol. 47, no. 12, pp. 903-909, 1935.

[3] S. Geltman, "Ionization of a model atom by a pulse of coherent radiation," Journal of Physics B, vol. 10, no. 5, article 019, pp. 831-840, 1977.

[4] S. Geltman, "Short-pulse model-atom studies of ionization in intense laser fields," Journal of Physics B, vol. 27, p. 1497, 1994.

[5] J. Z. Kamiński, "Stabilization and the zero-range models," Physical Review A, vol. 52, no. 6, pp. 4976-4979, 1995.

[6] Q. Su, B. P. Irving, C. W. Johnson, and J. H. Eberly, "Stabilization of a one-dimensional short-range modelatom in intense laser fields," Journal of Physics B, vol. 29, no. 23, pp. 5755-5764, 1996.

[7] S. Geltman, "Comment on "stabilization of a one-dimensional short-range model atom in intense laser fields"', Journal of Physics B, vol. 32, no. 3, pp. 853-856, 1999.

[8] E. P. Gross, "Structure of a quantizedvortex in boson systems," Nuovo Cimento, vol. 20, no. 3, pp. 454-477, 1961.

[9] E. P. Gross, "Hydrodynamics of a superfluid condensate," Journal of Mathematical Physics, vol. 4, no. 2, pp. 195-207, 1963.

[10] L. P. Pitaevskii, "Vortex lines in an imperfect Bose gas," Soviet Physics-JETP, vol. 13, p. 451, 1961.

[11] S. Geltman, "A critique on the misuse of the Gross-Pitaevskii equation," EPL, vol. 87, no. 1, p. 13001, 2009.

[12] A. Farrell and B. P. van Zyl, "S-wave scattering and the zerorange limit of the finite square well in arbitrary dimensions," Canadian Journal of Physics, vol. 88, no. 11, pp. 817-824, 2010.

[13] M. M. Nieto, "Existence of bound states in continuous $0<\mathrm{D}$ $<\infty$ dimensions," Physics Letters, Section A, vol. 293, no. 1-2, pp. 10-16, 2002.

[14] M. Abramowitz, I. A. Stegun et al., Handbook of Mathematical Functions, U S Government Printing Office, 1964.

[15] E. Fermi, Ric. Sci., vol. 7, p. 13, 1936.

[16] N. Levinsonson, Det kongelige Danske Videnskabernes Selskab Matematisk-Fysiske Meddelelser, vol. 25, no. 9, 1949.

[17] K. Wodkiewicz, "Fermi pseudopotential in arbitrary dimensions," Physical Review A, vol. 43, p. 68, 1991.

[18] R. Jackiw, Beg Memorial Volume, World Scientific, 1991.

[19] A. Cabo, J. L. Lucio, and H. Mercado, "On scale invariance and anomalies in quantum mechanics," American Journal of Physics, vol. 66, no. 3, pp. 240-246, 1998.

[20] I. Mitra, A. DasGupta, and B. Dutta-Roy, "Regularization and renormalization in scattering from Dirac delta potentials," American Journal of Physics, vol. 66, no. 12, pp. 1101-1109, 1998.

[21] S.-L. Neyo, "Regularization methods for delta-function potential in two-dimensional quantum mechanics," American Journal of Physics, vol. 68, p. 571, 2000.

[22] P. Gosdzinsky and R. Tarrach, "Learning quantum field theory from elementary quantum mechanics," American Journal of Physics, vol. 59, p. 70, 1991. 

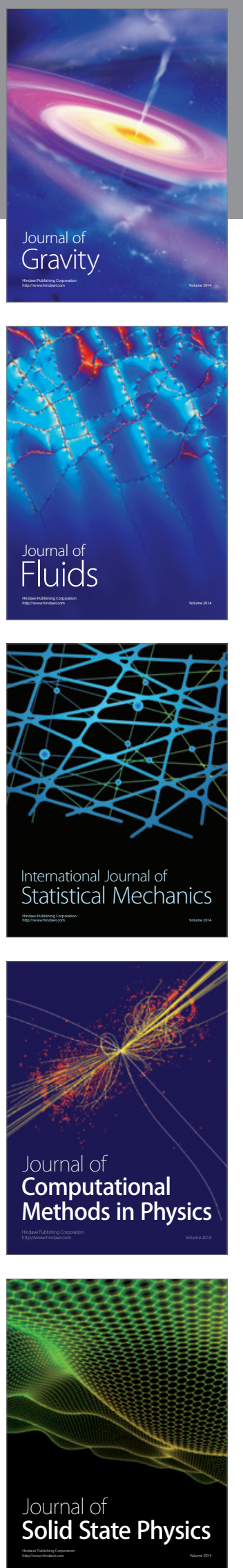

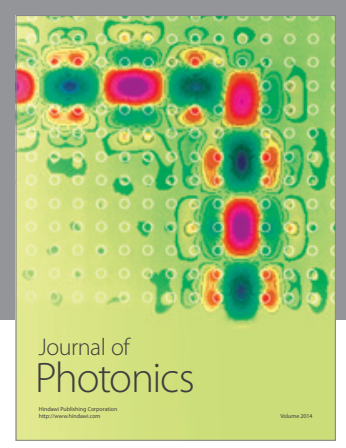

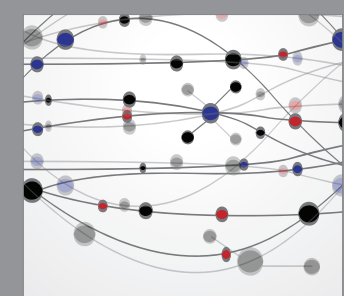

The Scientific World Journal
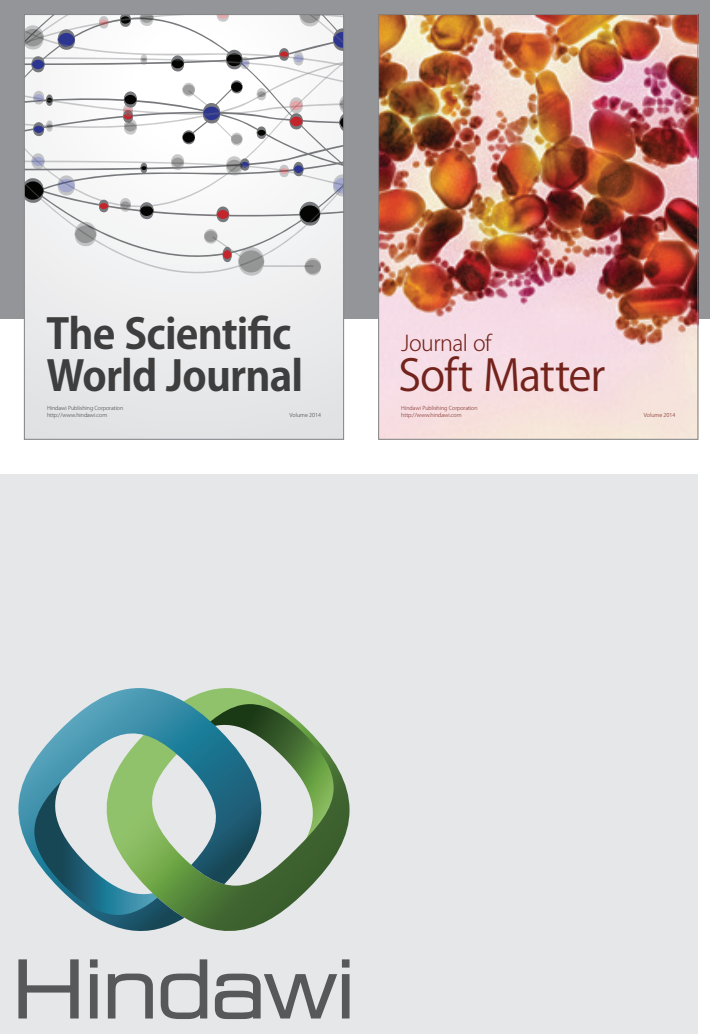

Submit your manuscripts at

http://www.hindawi.com
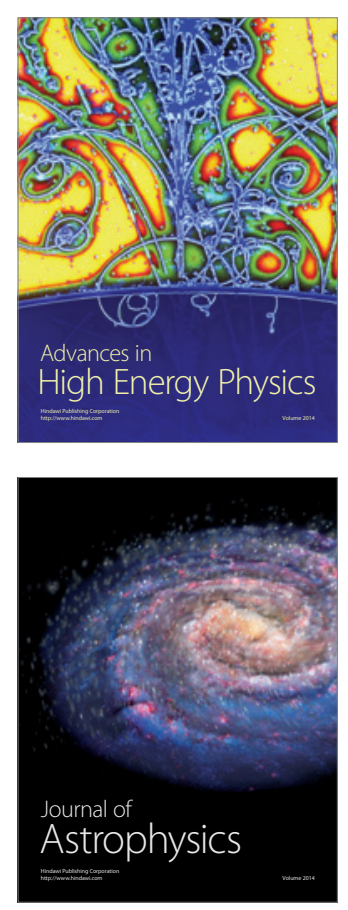
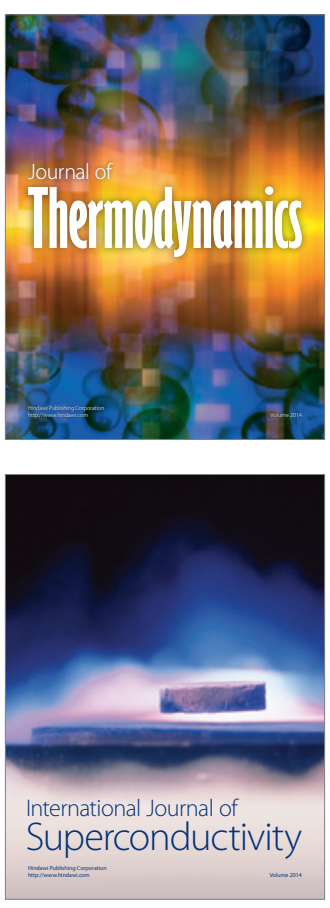
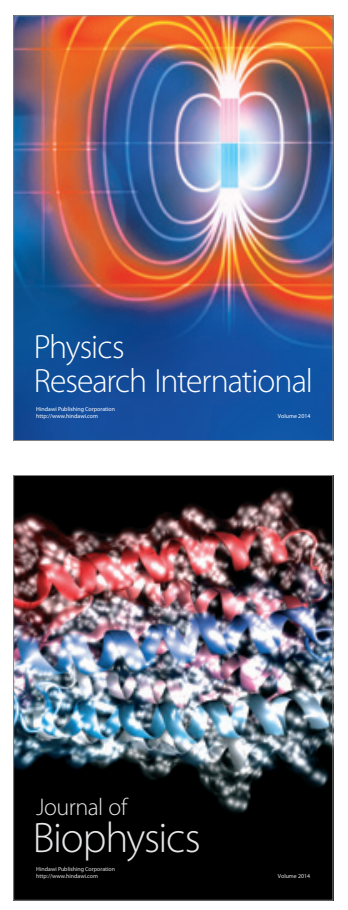
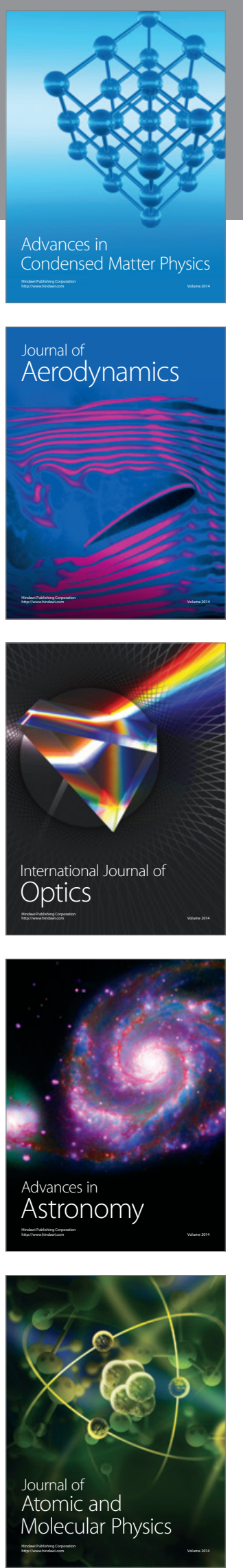